\title{
Statistical Properties of the Hybrid Radon-Fourier Technique
}

\author{
V.F. Leavers \\ Manchester School of Engineering, \\ Electrical Engineering Division, \\ Manchester University \\ v. leavers@man.ac.uk
}

\begin{abstract}
The hybrid Radon-Fourier technique has been proposed for the discrimination and tracking of deforming and compound targets. The current work investigates the technique's unique statistical properties which make it inherently robust with respect to performance. The Radon transform is used to generate the geometric signature waveform of the convex hull of the target, this then becomes the input to the Fourier Transform and the Fourier coefficients determine the parameters associated with the shape and motion of the target. Because, in general, relatively few points on the boundary of the object define the convex hull they will follow a Poisson distribution. In addition, for each point in the set of points defining the convex hull, there is a high probability that another neighboring point on or near the boundary may be substituted for that point with no significant effect on the performance of the algorithm. This means that the data may be extremely sparsely sampled without a significant degradation in the performance of the algorithm and with a corresponding reduction in the computational load. The theory is illustrated using 2-D data. The extension of the technique to 3-D data is discussed and is straightforward.
\end{abstract}

\section{Motivation}

The hybrid Radon-Fourier technique has been proposed as a solution to these machine vision tasks[1]. The current work investigates the technique's unique statistical properties which make it inherently robust with respect to performance. The remainder of this section deals with previous work concerning tracking and the ways in which it applies to the current work.

In the early literature concerning motion tracking the Fourier Transform was proposed as a potential means of tracking single objects in real-time[2]. However, the two suggested methods, global transformation of the image data[3] and segmentation of the object boundary prior to transformation[4], [5] were both seen to have drawbacks, which have precluded their use in any general form.

Paul Hough [6] first introduced the Hough transform, a special case of the Radon transform[7] in 1962. Since this time and as a consequence of its potential for grouping 
image data into perceptually meaningful features[8], the Hough transform has also been used to track rigid objects. For example, in [9]. edge image points are selected at random from two consecutive frames in a sequence of time-varying images. When the sampling produces two pairs of pixels that are similar, i.e. having the same translational motion, the similar translation is the estimated motion. Once detected, moving objects are segmented from the image. This procedure is repeated frame by frame. Other previous methods which use the Radon/Hough transform as part of a rigid object tracking algorithm do not use the image data directly[10]. Optic flow estimates are made using the frame by frame correspondences of each pixel of the image. The transformation process then groups the pixels moving with coherent motions into objects. In addition to the computational expense of the algorithm, the recovery of optic flow is an ill-posed problem and requires additional constraints in order to regularize the problem.

In order to track deforming objects, techniques that use Kalman filtering have been developed[11]. One of the most recent of these[12] uses measurements of gradient-based image potential and optical flow along the boundary of the object as the input data to the system. The method relies on the establishment of frame by frame correspondences between points in the image. It detects and rejects spurious measurements which are not consistent with previous estimation of image motion and is thus able to cope with relatively slowly varying deformations of the moving object.

There is currently no solution to the problem of tracking an unknown compound target that is relatively rapidly deforming as it moves. It is not possible to extend the available techniques of frame by frame model-based reasoning or inter-frame correspondences between points in the image in order to establish the parameters of motion of such an target. For this reason, the hybrid Fourier-Radon technique has been developed. The remainder of the paper is dedicated to illustrating the theory associated with the technique and its unique statistical properties.

\section{The Radon Transform}

The Radon Transform may be written in the convenient form:

$$
H(p, \xi)=\int_{-\infty}^{\infty} d \mathbf{x} F(\mathbf{x}) \delta(p-\xi \cdot \mathbf{x})
$$

Where $F(\mathbf{x})$ is a function defined on a domain $D$. In two dimensions, $\delta(p-\xi \cdot \mathbf{x})$, represents a delta function distribution situated along a line, $L$, with equation $p-\xi$. $\mathbf{x}=0$ where $\xi$ is a unit vector in the direction of the normal to that line and $p$ is the algebraic length of the normal. In three dimensions, $\delta(p-\xi \cdot \mathbf{x})$, represents a delta function distribution situated over a plane, where $\xi$ is still a unit vector but in the direction of the normal to the plane and $p$ is the algebraic length of the normal. For the purposes of illustration it is convenient to present the two dimensional case. The extension to three dimensions is accomplished by replacing the lines tangential to the shape in two dimensions with the planes tangential to the shape in three dimensions.

It is of particular interest to consider the case in which the general function $F(\mathbf{x})$ is replaced by a particular function $F_{D}(\mathbf{x})$, where 


$$
F_{D}(\mathbf{x})= \begin{cases}1, & \text { in } D ; \\ 0, & \text { otherwise. }\end{cases}
$$

This definition corresponds to the transformation of a binary image and this is the type of image transformed in the illustrations of the technique presented in this paper.

\section{Generating the Convex Hull}

The convex hull of a shape is defined as the minimum enveloping boundary, fitted to a shape outline, that is nowhere concave. Murakami et al[13] have shown that the upper or lower bounding curve of the Hough space can be used to determine the convex hull of the shape under detection. This is because the endpoints of the tangents to the convex hull are on lines, which are radially outermost with respect to a particular angle, the intersection of the cosine curves that they generate will form the bounding curve of the distribution in the transform space. Thus, it is the locus of the points forming the boundary of the transform plane which yield the equations of the tangents to the convex hull. Fig. 1 - bottom row, shows that if these tangents are plotted in image space they generate the convex hull of the shape under detection. However, we do not need to consider all of the possible tangents to the convex hull as shown in Fig. 1 as some of these will only pass through one image point. It is each intersection of the cosine curves that denotes the line joining two endpoints of a particular tangent and it is these points of intersection that define the corners or lobes of the convex hull as explained below.

In Fig. 1, the points marked A, B and $\mathrm{C}$ denote the image points that give rise to the three humps indicated on the bounding curve. Point $\mathrm{A}$ generates a cosine curve which is intersected by the cosine curve generated by point $\mathrm{B}$ and similarly for points $\mathrm{B}$ and $\mathrm{C}$. The minima are the points of intersection of the cosine curves. That is, the distance, $\alpha$, along the $\theta$ axis, between the second and third minima on the bounding curve in transform space gives the value of the angle $A B C$ in image space:

$$
A B C=\pi-\alpha
$$

Thus, in order to determine the convex hull of the shape under detection, it is only necessary to determine the locus of the points of intersection of the cosine curves on the boundary of the transform space. This holds true even when the shape is rounded because in the process of digitization any smoothly curving shape is expressed as a series of connected short lines. The convex hull of such a shape will be a many-sided, convex polygon.

\section{The Fourier-Series Expansion}

There are several methods of extracting the Fourier coefficients from boundary data. Using the radius vector method the boundary points of the object under detection are expressed in polar co-ordinates such that $r(\theta)$ represents the length of the radius vector directed from the origin to the boundary point and $\theta \epsilon[0,2 \pi]$ is the angle between the radius vector and the horizontal axis. The radius vector is thus a periodic function of the polar angle, $\theta$, and may be expanded into a Fourier-series of the form: 


$$
r(\theta)=\frac{a_{0}}{2}+\sum_{k=1}^{\infty}\left(a_{k} \cos (k \theta)+b_{k} \sin (k \theta)\right)
$$

where $k$ is a positive integer and the Fourier coefficients, $a_{0}, a_{k}$ and $b_{k}$, are given by:

$$
\begin{gathered}
a_{0}=\frac{1}{\pi} \int_{-\pi}^{\pi} r(\theta) d \theta \\
a_{k}=\frac{1}{\pi} \int_{-\pi}^{\pi} r(\theta) \cos (k \theta) d \theta \\
b_{k}=\frac{1}{\pi} \int_{-\pi}^{\pi} r(\theta) \sin (k \theta) d \theta
\end{gathered}
$$

The radius vector is also called the geometric signature waveform of the shape.

The necessary conditions for a function to be expanded as a Fourier series are as follows:

1. $r(\theta)$ must be defined and single valued throughout the periodic interval.

2. $r(\theta)$ must be continuous or have a finite number of discontinuities within the periodic interval.

3. $r(\theta)$ and $r^{\prime}(\theta)$ must be piecewise continuous in the periodic interval.

The first condition precludes the representation by a Fourier series of the boundary of an object with re-entrant features. Many objects which we might wish to track have such features. The second condition means that only a closed boundary may be represented by a Fourier series and this is often not possible with noisy, incomplete or occluded image data. In particular, a compound target made up of a group of objects will not have a closed boundary. On a practical note, the input to the Fast-Fourier-Transform algorithm requires the input data to be an evenly-spaced, binary-number of points and this condition has the same disadvantages as the first and second conditions above.

\section{The Radon-Fourier Connection}

The current work is novel in that it uses the upper or lower bounding curve of the Radon/Hough Transform, which, in the case of the normal parameterization[14], is also the geometric signature waveform of the convex hull of the shape under detection, as input to the onedimensional Fourier Transform.

Inspection of Fig. 2 shows that, for a circle centered on the origin and of radius 30 pixels, the locus of the bounding curve of the Radon Transform corresponds to the geometric signature waveform. See Fig. 2 - bottom right. Equation 4 shows that $a_{0}$ is simply twice the mean value of $r(\theta)$; in this case, the diameter of the circle. The $a_{0}$ coefficient can be scaled but will not be affected by either rotation or translation.

If the first harmonic is considered, $k=1$, then inspection of the RHS's of equations 5 and 6 show that each is simply the definition of the Cartesian co-ordinates of the centroid of the shape, $\left(x_{10}, y_{10}\right)$, respectively. If Equation 3 is expressed in the form:

$$
r(\theta)=\frac{a_{0}}{2}+\sum_{k=1}^{\infty} A_{k} \cos \left(k \theta-\phi_{k}\right)
$$


where: $A_{k}=\sqrt{{a_{n}{ }^{2}+b_{n}{ }^{2}}}$ is the amplitude and $\phi_{k}$ is the phase of the $k t h$ harmonic, then the amplitude and the phase of the first harmonic can be used to determine the coordinates of the centroid. That is, if the circle is displaced such that it is no longer centered on the origin then the effect of that translation, $\left(x_{1_{0}}, x_{2_{0}}\right)$, on the distribution in transform space is given by:

$$
R(p, \theta) \mapsto R\left(p+\left(x_{1_{0}}{ }^{2}+x_{2_{0}}{ }^{2}\right)^{\frac{1}{2}} \cos \left(\theta-\tan ^{-1}\left(\frac{x_{2_{0}}}{x_{1_{0}}}\right)\right), \theta\right)
$$

On the middle row of Fig.2, the circle has been displaced by $(30,30)$. The corresponding calculated Fourier parameters are: $A_{1}=42$ and $\phi_{1}=45^{\circ}$. On the bottom row the circle has been displaced by $(-30,-30)$. The corresponding calculated Fourier parameters are: $A_{1}=42$ and $\phi_{1}=-90^{\circ}$. Thus, the $A_{1}$ and $\phi_{1}$ parameters describing the first harmonic are not functions of the shape but of the displacement of the centroid of the shape from the origin of the image.

It is possible to create various shapes by the addition of higher order harmonics to the geometric signature waveform. Fig.3 - top row, shows an example of adding the second harmonic in order to create an elliptical shape. The middle row shows the elliptical shape displaced by $(30,30)$.

Any convex hull can be represented by a geometric signature waveform which will have a canonical Fourier description generated such that its centroid is co-incident with the origin. In this case each of the $\phi_{k}$ in equation 7 will have a value of $\phi_{k_{o}}$ depending on the starting point used to generate $r(\theta)$.

If the shape is rotated about the centroid by an angle, $\phi_{r}$, this causes a shift in the $\theta$ direction only. The original and new transforms are related by:

$$
R(p, \theta) \mapsto R\left(p, \theta+\phi_{r}\right)
$$

Substituting for $\theta$ in equation 7 :

$$
\phi_{k} \mapsto \phi_{k_{0}}-2 \phi_{r}
$$

On the bottom row of Fig.3 is the image of the elliptical shape rotated by an angle of $45^{\circ}$ about its centroid and then shifted by $(30,30)$. From the canonical value $\phi_{20}$ and the calculated value of $\phi_{2}$ when the shape is rotated we can determine the angle of rotation to be $45^{\circ}$. Thus the angle of rotation about the centroid can be deduced using any of the calculated $\phi_{k}$, for $k>2$.

\section{Statistical Properties of the Technique}

The computational load associated with calculation of both the Radon and the Fourier transform can be prohibitive in the case of real-time applications. Given the proposed combination of both transforms, it is of interest to consider ways in which the data may be sampled and to quantify the effect that such sampling may have on the performance of the algorithm.

In general, relatively few points on the boundary of the object define the convex hull: thus, it is expected that they will follow a Poisson distribution given by: 


$$
p_{X}(x)=\frac{e^{-\mu} \mu^{x}}{x !}
$$

In addition, for each point in the set of points defining the convex hull, there is a high probability that another neighboring point on or near the boundary may be substituted for that point with no significant effect on the performance of the algorithm. Small random perturbations in the position of each individual point in a set of points will tend to cancel out other perturbations such that the overall effect is within the range of errors introduced by digitizing the image or in calculating the parameters. This means that there are a combinatorial number of sets of points which may be used to define the convex hull without a significant degradation in the performance of the algorithm. The expectation is that the data may be extremely sparsely sampled without a corresponding reduction in the performance of the algorithm. What follows is a quantitative investigation of the statistical properties of the technique using real data.

\subsection{Quantitative Analysis of Statistical Properties}

In order to investigate quantitatively the statistical properties of the technique it is first necessary to ascertain how many points on the boundary of an object define the convex hull. This can be done by determining the number, $k$, of intersections of cosine curves on the bounding curve in the transform space. The proportion of such points within the edge image data is given by: $p=k / N$; where $N$ is the total number of edge points.

If we randomly remove $n$ of the edge image points, without replacement, then for $\mu=n p$, the probability of removing all $k$ points in a sample of size $n$, where $n \geq k$, may be expressed as:

$$
P(k)=1-\sum_{x=0}^{k-1} \frac{e^{-\mu} \mu^{x}}{x !}
$$

In order to test the validity of this hypothesis the following experiment was carried out using the real image data. A photo of three starship objects was taken with a flash camera using a black and white film. To segment the objects from their background a median filter was applied to the image to remove the bright spots introduced by the flash. This image was then averaged to remove the uneven background lighting effect. The processed image was edge detected and thresholded to produce the data required as input to the Radon transform. Following the transformation, the coordinates of the bounding curve were determined and used as input to the FFT. The Fourier coefficients were then used to calculate the $(x, y)$ coordinates of the centroid of the convex hull of the formation, see Fig. 4, top right. The calculated values of the centroid coordinates are rounded to integers because the smallest unit of measurement in a digitized image is one pixel. This introduces a digitization error or \pm 1 in each of the calculated values.

The transformation of the compound target produces a bounding curve composed of three intersecting cosine curves (see Fig. 4, bottom left). What this means is that three points in the edge image are defining the convex hull of the formation. Clearly the convex hull has a more detailed shape, however, the quantisation in the transform space is not able to resolve this detail. 
A percentage of the edge points was randomly removed from the data set of edge points without replacement. This was repeated 100 times each, for a number of percentage values in the range [10\%-99\%]. The sequence of random numbers used had $2^{1492}$ numbers before any sequence of numbers would repeat. In addition, each trial was made statistically independent of any other trial by using a fresh seed to generate the first random number in each series of 100 trials and an additional randomizing shuffle to generate a list of seeds for each of the 100 trials in any given series.

The standard deviation in the calculated centroid coordinates was chosen as a measure of the performance of the algorithm. Thus, if the Poisson distribution was the only factor effecting the performance of the algorithm as points are randomly removed without replacement, then we would expect the degradation in the calculated values of the standard deviation to vary according to the form of the curve generated by Equation 12 with a value of $k=3$.

Fig. 5 shows the standard deviation in the 100 calculated values of the $x$ coordinate of the centroid at each point in the range of percentage points tested (each point is ringed); also shown is the curve generated using Equation 12 for a value of $k=3$. We see that the shapes of the two curves are different. The difference is explained by the effect of the combinatorial number of sets of points able to generate a good estimate of the centroid. A dotted line along the value of 1 represents the digitization error. The standard deviation does not exceed the digitization error until the $97 \%$ point is passed. At the $99 \%$ point the standard deviation is still relatively small: a value of less than two pixels. For this particular image the size of the smallest circumventing circle (shown in Fig. 4, top right) is 280 pixels. This gives an error in estimating the $x$ coordinate of the centroid of $\pm 0.7 \%$ of the size of the target at the 99\% removal point. A similar result was obtained for the $y$ coordinate of the centroid.

Thus, instead of the standard deviation increasing to exceed the digitization error at the $70 \%$ removal point, (as would be the case if the results were attributable simply to the Poisson statistics of the data), we see that the performance does not begin to degrade until the $97 \%$ removal point has been reached. In addition, the degradation in performance is relatively small even at the $99 \%$ removal point. To illustrate this, the scatter in the $(x, y)$ coordinates resulting from 500 independent trials at the $95 \%$ and the $99 \%$ removal points was determined. The maximum scatter was $1.2 \%$ and $2.8 \%$ of the target size in the case of the $95 \%$ and $99 \%$ removal points respectively.

\section{Conclusion}

The hybrid Radon-Fourier technique has been proposed for the discrimination and tracking of deforming and compound targets. The current work investigated the technique's unique statistical properties. The Radon transform was used to generate the geometric signature waveform of the convex hull of the target, this then became the input to the Fourier Transform and the Fourier coefficients determined the parameters associated with the shape and motion of the target. In general, relatively few points on the boundary of the object define the convex hull; thus, they will follow a Poisson distribution. In addition, for each point in the set of points defining the convex hull, there is a high probability that another neighboring point on or near the boundary may be substituted for that point; it was shown that, because of this, the data may be extremely sparsely sampled without 
a significant degradation in the performance of the algorithm and with a corresponding reduction in the computational load. The theory was illustrated using 2-D data. The extension of the technique to 3-D data was discussed and is straightforward.

\section{References}

[1] Leavers V.F. Motion Monitoring, U.K. patent application 0000811.0, January 2000.

[2] Huang T.S., Image sequence analysis. Springer Verlag, ISBN 0-387-10919-6, 1981.

[3] Pearson J.J., Hines D.C., Golosman S. and Kuglin C.D., Video image correlation processor. SPIE Vol 119 Application of Digital Image Processing, pp 197-205, 1977.

[4] Wallace T., Mitchell O. and Fukunaga K., 3D shape analysis using local shape descriptors. IEEE Conf. on Pattern Recognition and Image Processing, pp 292-299, 1979.

[5] Lin C.C. and Chellappa R., Classification of partial 2-D shapes using Fourier Descriptors IEEE TRANS PAMI, Vol 9(5), pp 686-690, 1987.

[6] Hough P.V.C. Method and means for recognizing complex patterns. U.S. Patent 3069654, 1962

[7] Deans S.R. Hough transform from the Radon transform. IEEE Trans. Pattern Analysis and Machine Intelligence. Vol. PAMI-3, No., March 1981.

[8] Leavers V.F., Survey: Which Hough Transform?, Computer Vision Image Processing and Graphics, Vol 58(2), pp 250 - 264, 1993.

[9] Kalviainen, Motion detection using the randomized Hough Transform: exploiting gradient information and detecting multiple moving objects. IEE Proc.-Vis. Image Signal Process., Vol 143(6), pp 361-369, December 1996.

[10] Bober M. and Kittler J., Estimation of complex multimodal motion: an approach based on robust statistics and Hough Transform. Image and Vision Computing, Vol 12(10), pp 661-668, 1994.

[11] Terzopoulos D. and Szeliski R., Tracking with Kalman snakes. Active Vision, Blake A. and Yuille A. eds., pp 3-20, MIT Press, 1992

[12] Peterfreund N., Robust tracking of position and velocity with Kalman Snakes IEEE TRANS PAMI, Vol 21(6), pp 564-569, June 1999.

[13] Murakami K., Koshimizu H. and Hasegawa K., An algorithm to extract convex hull in Hough space. Proc. 4th Int. Conf. on Pattern Recognition, 1988

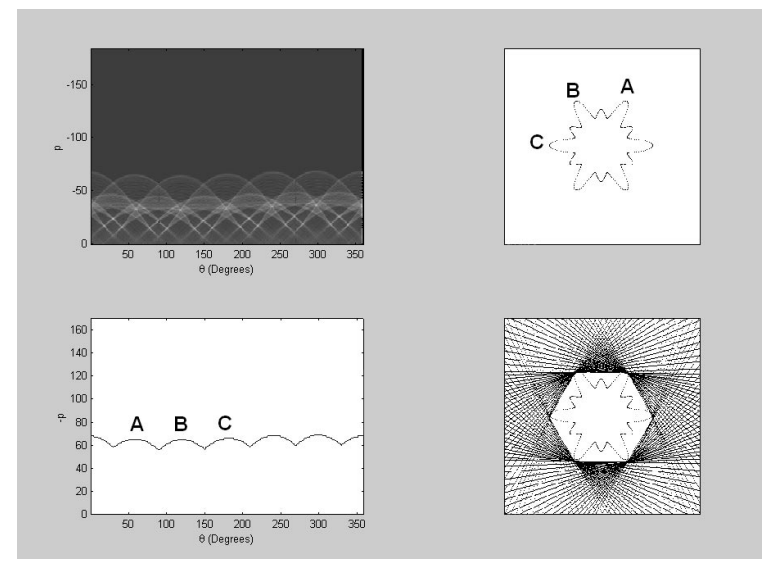

Fig. 1 Top: Intensity map of transform plane generated by shape on the right Bottom: Bounding curve and corresponding tangents in image space 

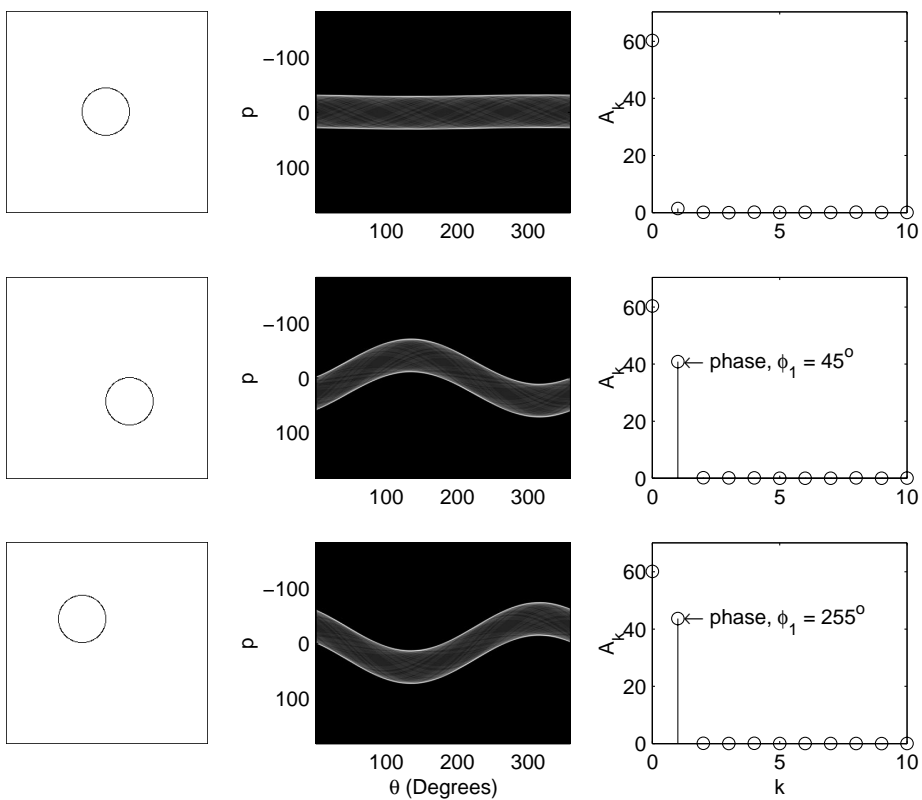

Fig. 2 Left: Images of circles Middle: Intensity maps of transform planes Right: Fourier coefficients, $A_{k}$
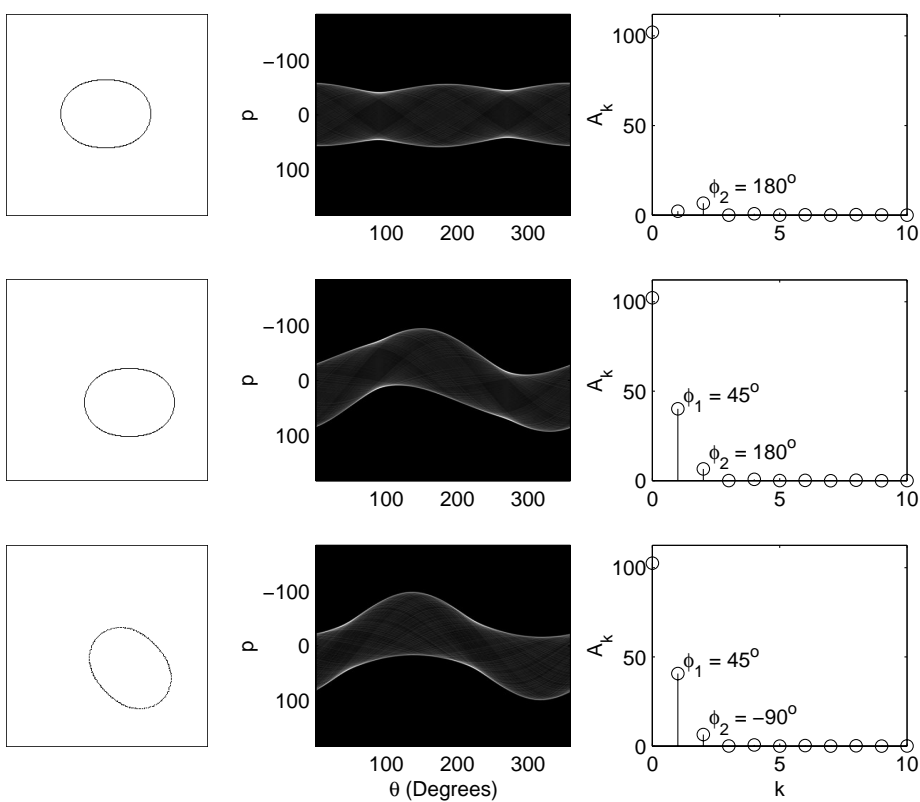

Fig. 3 Left: Images of ellipses Middle: Intensity maps of transform planes Right: Fourier coefficients, $A_{k}$ 

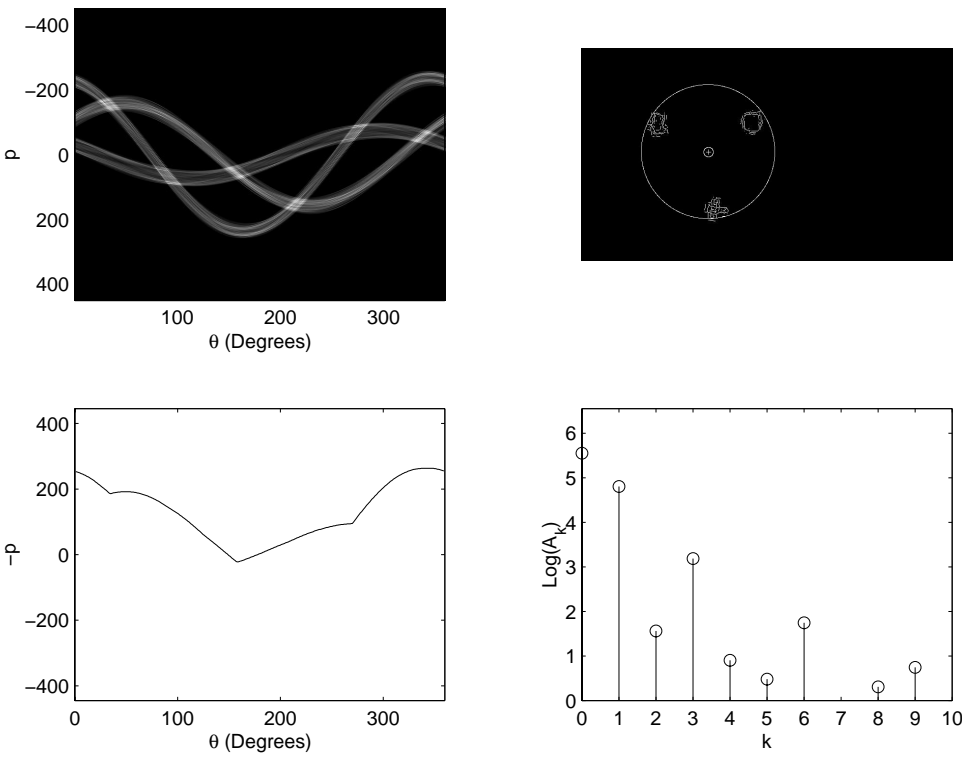

Fig. 4 Top Left: Radon Transform Right: Binary Edge Image of Formation Bottom Left: Upper bounding curve of transform space Right: Fourier Coefficients

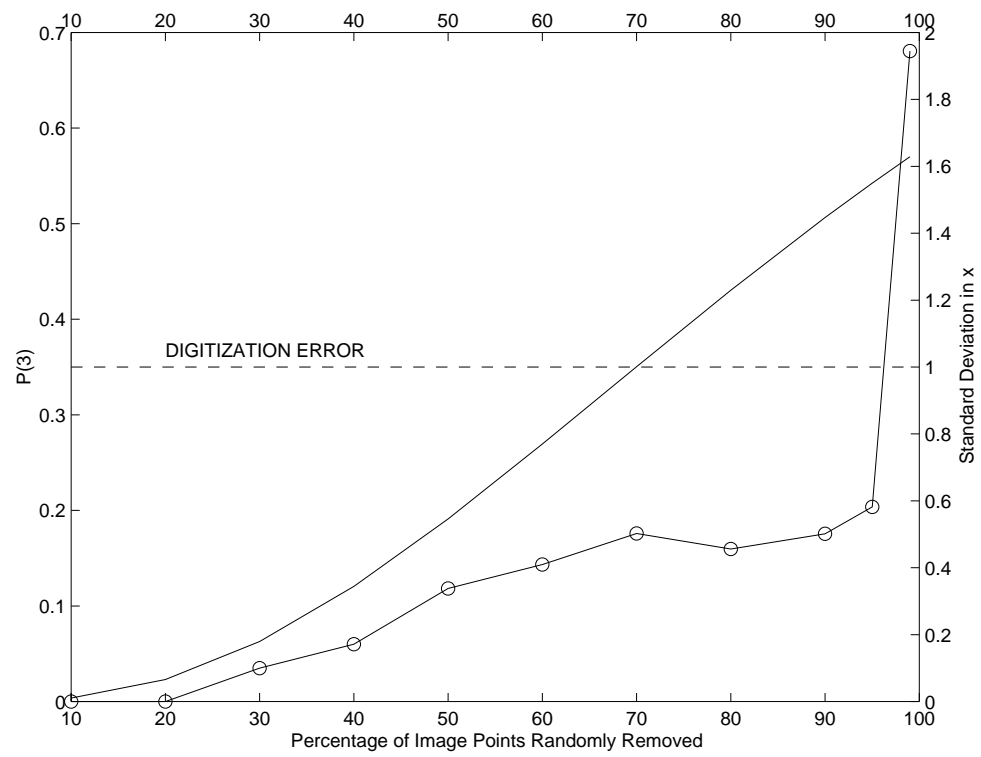

Fig. 5 Standard deviation in calculating x coordinate of centroid 